\title{
T Dwarf Photometric Variability
}

\author{
Etienne Artigau, Daniel Nadeau, René Doyon \\ Département de physique, Université de Montréal, C.P. 6128, Succ. A, \\ Montréal, QC, Canada HзC зJ7
}

\begin{abstract}
We report on a $J$ and $H$ photometric variability survey of $10 \mathrm{~T}$ dwarfs. Four brown dwarfs in the sample show significant $J$ band variability at the $99 \%$ confidence level or higher. The amplitude of variation ranges from 2 to $17 \%$. Our data show evidence of variability for late as well as early $\mathrm{T}$ dwarfs.
\end{abstract}

\section{Introduction}

Photometric variability of $\mathrm{M}$ and $\mathrm{L}$ dwarfs has been observed in the $I$ band (Bailer-Jones \& Mundt 2001, Gelino et al. 2002, and references therein). The observations presented here extend the search for photometric variability to $\mathrm{T}$ dwarfs. As $\mathrm{T}$ dwarfs are extremely faint in the $I$ band, observations were performed in the $J$ and $H$ bands. The mechanisms involved in brown dwarf variability are still unknown and their nature may vary with atmospheric temperature. Evolving cloud patterns are prime candidates to explain brown dwarf variability. Such atmospheric features are conspicuous in the atmospheres of the solar system giant planets. If present on BDs, it will be important to take them into account for the detailed modelling of BD atmospheres. It is noteworthy that many objects detected as variable do not show a periodic light curve, and thus hint that surface features covering at least a few percent of the visible disk appear and disappear one timescales of a few hours or less (Bailer-Jones \& Mundt, 2001).

\section{Target Selection, Observations and Data Reduction}

The $\mathrm{T}$ dwarfs observed for photometric variability were selected from those observable for several hours from the northern hemisphere $(\delta>-5)$ and having at least two bright stars within a field of view of $2^{\prime} \times 2^{\prime}$. The sample spawns most of the spectral range of $\mathrm{T}$ dwarfs, with objects from $\mathrm{T} 0$ to $\mathrm{T} 7.5$. Observations were obtained during four observing runs, in $2001 \mathrm{March}$, April, December and 2002 March at the $\mathrm{f} / 8$ focus of the $1.6 \mathrm{~m}$ telescope of the Observatoire du MontMégantic with the MONICA (Nadeau et al. 1994) infrared camera. Differential aperture photometry was performed using a 2 FWHM diameter aperture. 


\section{Results and Discussion}

For all objects, photometric measurements were obtained on at least two nights. Night-to-night variability was searched for using the Student's t-test. All results are summarized in Table 1. Four out of the $10 \mathrm{~T}$ dwarfs observed show significant $J$ band photometric variability: SD1254, 2M1237, 2M0727 and 2M1217 have spectral type $\mathrm{T} 2, \mathrm{~T} 6.5, \mathrm{~T} 7$ and $\mathrm{T} 7.5$ respectively.

Burgasser et al. 2002a suggest that the $J-K_{s}$ color is a good indicator of the dust patch coverage in a $\mathrm{T}$ dwarf atmosphere. $\mathrm{T}$ dwarfs without dust patches have a $J-K_{s} \sim 0$, and a brown dwarfs with an atmosphere totally covered with dust clouds have a $J-K_{s} \sim 2$. SD1254 with a $J-K_{s} \sim 1$ has a $40 \%$ dust patch coverage. The detection of variability on SD1254 is consistent with the prediction by Burgasser et al. (2002a); the light curve for SD1254 is shown on Figure 1. Nevertheless we see that the T6.5, T7 and T7.5 dwarfs detected as variable in this study have a $J-K_{s} \sim 0$. This suggests that evolving dust patches is not the only mechanism involved in $\mathrm{T}$ dwarf variability.

Slight variability at the $\sim 30$ millimagnitude level in the $J$ band has been detected for 2M1237, the only known $\mathrm{T}$ dwarf with $\mathrm{H}_{\alpha}$ emission. Burgasser et al. $(2000,2002 b)$ found no clear $\mathrm{H}_{\alpha}$ variability for this object and no evidence of $J$ band variability, but the error bars in their measurements preclude the detection of variability at the level detected here. A dedicated simultaneous $\mathrm{H}_{\alpha}$ and near-infrared monitoring would be needed to determine if, for this object, variability is related to magnetic activity.

The sparse time sampling of our observations allow only the setting of a lower limit on the prevalence and amplitude of variability in T dwarfs. More observations are needed to further quantify the variability timescales. Results from the CLOUDS project (Goldman, in these proceedings) confirm the variability of SD1254 and 2M1237 and give information on variability timescales.

This work is supported in part through grants from the Natural Sciences and Engineering Research Council, Canada and from FQRNT, Québec.

\section{References}

Bailer-Jones, C. A. L. \& Mundt, R., 2001, A\&A, 367, 218

Burgasser, A. J., Wilson, J. C., Kirkpatrick, J. D., 2000, AJ, 120, 1100

Burgasser, A. J., Kirkpatrick, J. D., Reid, I. N., Liebert, J., Gizis, J. E. \& Brown, M. E., 2000, AJ, 120, 473

Burgasser, A. J., Marley, M. S., Ackerman, A. S., Saumon, D., Lodders, K., Dahm, C. C., Harris, H. C. \& Kirkpatrick, J. D., 2002a, ApJ, 576 1, L151 
Burgasser, A. J., Liebert, J., Kirkpatrick, J. D. \& Gizis, J. E., 2002b, AJ, 123, 2744

Gelino, C. R., Marley, M. S., Holtzman, J. A., Ackermanm A.S. \& Lodders, K., (astro-ph/0205305)

Nadeau, D., Murphy, D. C., Doyon, R. \& Rowlands, N. 1994, PASP, 106, 909

TABLE 1

Differential photometry

\begin{tabular}{|c|c|c|c|c|}
\hline Object & Type & Epoch & $\begin{array}{c}J \\
\text { (mmag) }\end{array}$ & $\begin{array}{c}H \\
(\mathrm{mmag})\end{array}$ \\
\hline SDSSp J042348.6-041403 & $\mathrm{T} 0$ & $\begin{array}{l}2254 \\
2255\end{array}$ & $\begin{array}{r}0 \pm 3 \\
21 \pm 8\end{array}$ & \\
\hline SDSSp J125453.9-012247 & $\mathrm{T} 2$ & $\begin{array}{l}2011 \\
2016\end{array}$ & $\begin{array}{r}0 \pm 4 \\
45 \pm 2\end{array}$ & $\begin{array}{r}0 \pm 7 \\
23 \pm 4\end{array}$ \\
\hline SDSSp J102109.7-030420 & $\mathrm{T} 3$ & $\begin{array}{l}2014 \\
2015\end{array}$ & $\begin{array}{l}0 \pm 6 \\
1 \pm 7\end{array}$ & $\begin{array}{c}0 \pm 8 \\
11 \pm 14\end{array}$ \\
\hline SDSSp J092615.4+584720 & $\mathrm{T} 4.5$ & $\begin{array}{l}2250 \\
2251 \\
2253 \\
2254\end{array}$ & $\begin{array}{r}0 \pm 6 \\
8 \pm 9 \\
3 \pm 5 \\
19 \pm 6\end{array}$ & $\begin{array}{l}0 \pm 9 \\
4 \pm 9 \\
9 \pm 12\end{array}$ \\
\hline 2MASSI J104753.9+212423 & $\mathrm{T} 5$ & $\begin{array}{l}1970 \\
1972\end{array}$ & $\begin{aligned} 0 & \pm 10 \\
-11 & \pm 4\end{aligned}$ & \\
\hline 2MASSI J225418.8+312349 & $\mathrm{T} 5$ & $\begin{array}{l}2250 \\
2251 \\
2253 \\
2254\end{array}$ & $\begin{aligned} 0 & \pm 12 \\
22 & \pm 12 \\
16 & \pm 7 \\
-11 & \pm 17\end{aligned}$ & \\
\hline SDSSp J162414.4+002915 & $\mathrm{T} 6$ & $\begin{array}{l}2014 \\
2015 \\
2016\end{array}$ & $\begin{array}{r}0 \pm 4 \\
-14 \pm 7 \\
8 \pm 7\end{array}$ & $\begin{array}{r}0 \pm 7 \\
6 \pm 8 \\
19 \pm 9\end{array}$ \\
\hline 2MASSI J123739.2+652615 & T6.5 & $\begin{array}{l}1970 \\
1972\end{array}$ & $\begin{array}{r}0 \pm 8 \\
-14 \pm 5\end{array}$ & \\
\hline & & $\begin{array}{l}1976 \\
2017\end{array}$ & $15 \pm 10$ & $\begin{array}{r}0 \pm 5 \\
-24 \pm 6\end{array}$ \\
\hline 2MASSI J072718.2+171001 & $\mathrm{T} 7$ & $\begin{array}{l}2255 \\
2371\end{array}$ & $\begin{array}{c}0 \pm 6 \\
91 \pm 12\end{array}$ & \\
\hline 2MASSI J121711.1-031113 & $\mathrm{T} 7.5$ & $\begin{array}{l}1972 \\
2374\end{array}$ & $\begin{array}{r}0 \pm 13 \\
176 \pm 13\end{array}$ & \\
\hline
\end{tabular}

${ }^{a} \mathrm{JD}-2450000$ 


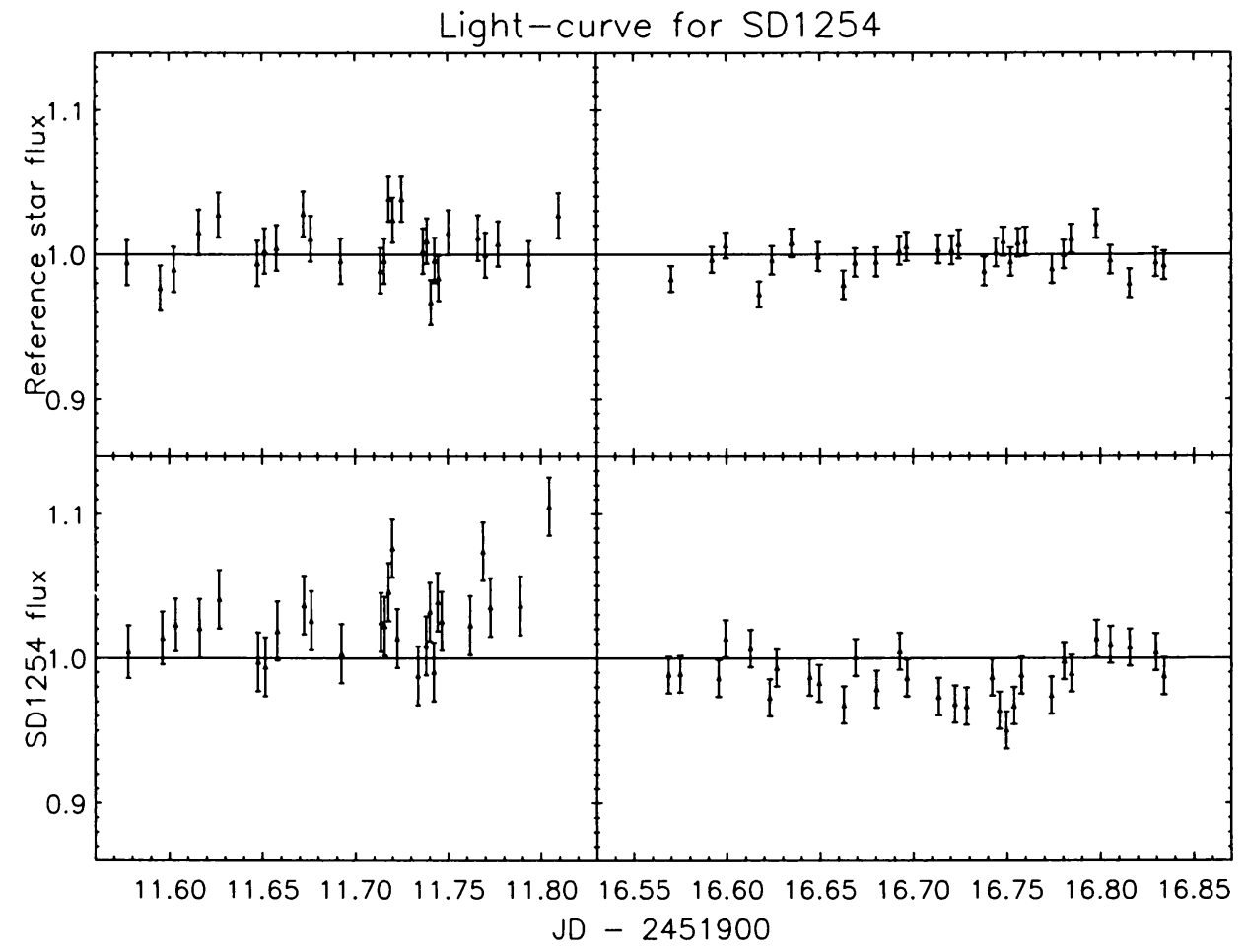

Figure 1. $J$ band light curve for SD1254 (lower panels) and a nearby reference star (top panels). A Student's t-test shows that there is significant variations between nights for SD1254. 